\title{
A homogeneous cell-based bicistronic fluorescence assay for high-throughput identification of drugs that perturb viral gene recoding and read-through of nonsense stop codons
}

TONY S. CARDNO, ELIZABETH S. POOLE, SUNEETH F. MATHEW, RYAN GRAVES, and WARREN P. TATE

Department of Biochemistry, University of Otago, Dunedin 9054, New Zealand

\begin{abstract}
Recoding mechanisms are programmed protein synthesis events used commonly by viruses but only very rarely in cells for cellular gene expression. For example, HIV-1 has an absolute reliance on frameshifting to produce the correct ratio of key proteins critical for infectivity. To exploit such recoding sites as therapeutic targets, a simple homogeneous assay capable of detecting small perturbations in these low-frequency $(<5 \%)$ events is required. Current assays based on dual luciferase reporters use expensive substrates and are labor-intensive, both impediments for high-throughput screening. We have developed a cellbased bifluorophore assay able to measure accurately small recoding changes $(<0.1 \%)$ with a high $Z^{\prime}$-factor in 24 - or 96 -well formats that could be extended to 384 wells. In cases of nonsense mutations arising within coding regions of genes, the assay is suitable for assessing the potential of screened compounds to increase read-through at these nonprogrammed stop signals of variable termination efficiency.
\end{abstract}

Keywords: bicistronic fluorescence; recoding assay; high-throughput screening; nonsense codon mutations

\section{INTRODUCTION}

Although an essential function of the genetic code is maintenance of the mRNA reading frame by the ribosome during protein synthesis, programmed recoding events (Gesteland et al. 1992) do occur rarely. For example, in response to cis elements in the mRNA, the ribosome can change frame by slipping forward $(+1)$ or backward $(-1)$ one nucleotide. While bioinformatic analyses suggest that frameshift sites are not uncommon in the eukaryotic genome (Jacobs et al. 2007), the mechanism is likely to be rare in the expression of cellular genes. It is, however, relatively common in viruses (Poole et al. 2004). In HIV frameshifting facilitates a precise ratio of structural Gag proteins to enzymatic Pol proteins (Shehu-Xhilaga et al. 2001). Frameshifting occurs at a "slippery" heptamer sequence, XXXYYYZ, in the viral RNA that is invariant in all sequenced isolates of HIV-1 so far (Biswas et al. 2004). The

Reprint requests to: Warren P. Tate, Department of Biochemistry, University of Otago, P.O. Box 56, Dunedin 9054, New Zealand; e-mail: warren.tate@stonebow.otago.ac.nz; fax: 64-3-479-7866.

Article published online ahead of print. Article and publication date are at http://www.rnajournal.org/cgi/doi/10.1261/rna.1586709. frameshift site is followed by a GGG codon at the start of a highly conserved extended stem-loop sequence that modulates the efficiency of the noncanonical event (Dulude et al. 2002). The same mechanism has been identified in plant, yeast, and bacterial viruses (Farabaugh 1996) and in other human viruses such as SARS-CoV (Dos Ramos et al. 2004).

Read-through of stop codons is a recoding event used by cells and viruses (Bertram et al. 2001) where amino acids are incorporated into a growing polypeptide through tRNA-mediated decoding. It is programmed during the synthesis of cellular proteins containing selenocysteine at specific UGA stop codons (Small-Howard and Berry 2005). Premature termination codon (PTC) mutations are reported to be the cause of at least 1800 human genetic diseases (Kellermayer 2006). Modeling PTC termination signals, and finding drugs that enhance their read-through would provide significant benefit.

Assays to measure accurately frameshift and readthrough recoding events have most recently used dual Firefly (Photinus pyralis) and Sea pansy (Renilla reniformis) luciferase reporter genes. The downstream reporter is expressed only as a fusion protein with the upstream reporter if the recoding event occurs (Grentzmann et al. 1998; 
Harger and Dinman 2003). The luciferase reporters have specific substrates with the test sample split into two aliquots and one luciferase activity measured in each fraction. With more expensive reagents, the first luciferase activity is assessed and quenched and the second luciferase measured within the same sample. This type of in vitro assay has signal enhancement through the catalytic activity of the enzymes, but it is less accessible to most researchers for larger screens because of cost. In contrast, fluorophore reporters do not require expensive substrates and can be detected directly in unfractionated samples in vivo and in vitro using only excitation and emission wavelengths of light that can be read in simple spectrophotometers. The challenge with this technology is to gain sufficient specificity and sensitivity so that low frequency events and their modulations can still be detected despite the lack of catalytic enhancement. While bicistronic fluorophore reporters have been described that use bacterial systems (Dulude et al. 2008, Liao et al. 2008), so far this technology has not been applied successfully for highthroughput screening (HTS) in an in vivo mammalian system. For this purpose, we have developed and modeled for the first time a sensitive bicistronic fluorophore assay in a microplate format, and show that it can detect very small changes accurately and reproducibly in rare genetic recoding events and within the range that might be induced by drugs in a large-scale compound screen.

\section{RESULTS}

\section{Orientation and spacing of reporter fluorophores}

First, we selected the best combination of fluorophores from the green fluorescent protein (GFP) color variants as the two reporters for the bicistronic constructs. They were enhanced GFP (EGFP), derived from the jellyfish Aequorea victoria GFP but codon optimized for mammalian cells, and the rapidly maturing red variant DsRed.T4 (also the more sensitive red derivative, tandem dimer tomato, tdTomato) (Shaner et al. 2004) derived from the coral Discosoma (Bevis and Glick 2002). Both green and red fluorophores have strong fluorescence with their excitation and emission maxima well separated (excitation: $485 \mathrm{~nm}$ and $555 \mathrm{~nm}$ respectively; emission: $507 \mathrm{~nm}$ and $586 \mathrm{~nm}$ respectively) (Fig. 1A). While the relative brightness of DsRed.T4 is reported to be less than that of EGFP (http:// www.microscopyu.com/articles/livecellimaging/fpintro.html), serial cell lysate dilutions of each fluorophore expressed separately showed that DsRed.T4 gave a better signal to background ratio than EGFP over at least a 2000 -fold range (Fig. 1B). This implied that DsRed.T4 could be the better choice for the low frequency output of the recoding event. To test whether EGFP and DsRed.T4 fluorescence could be quantified independently without interference from the companion fluorophore in the same cell lysate, fluorescence intensities were measured in lysates of EGFP spiked with
A
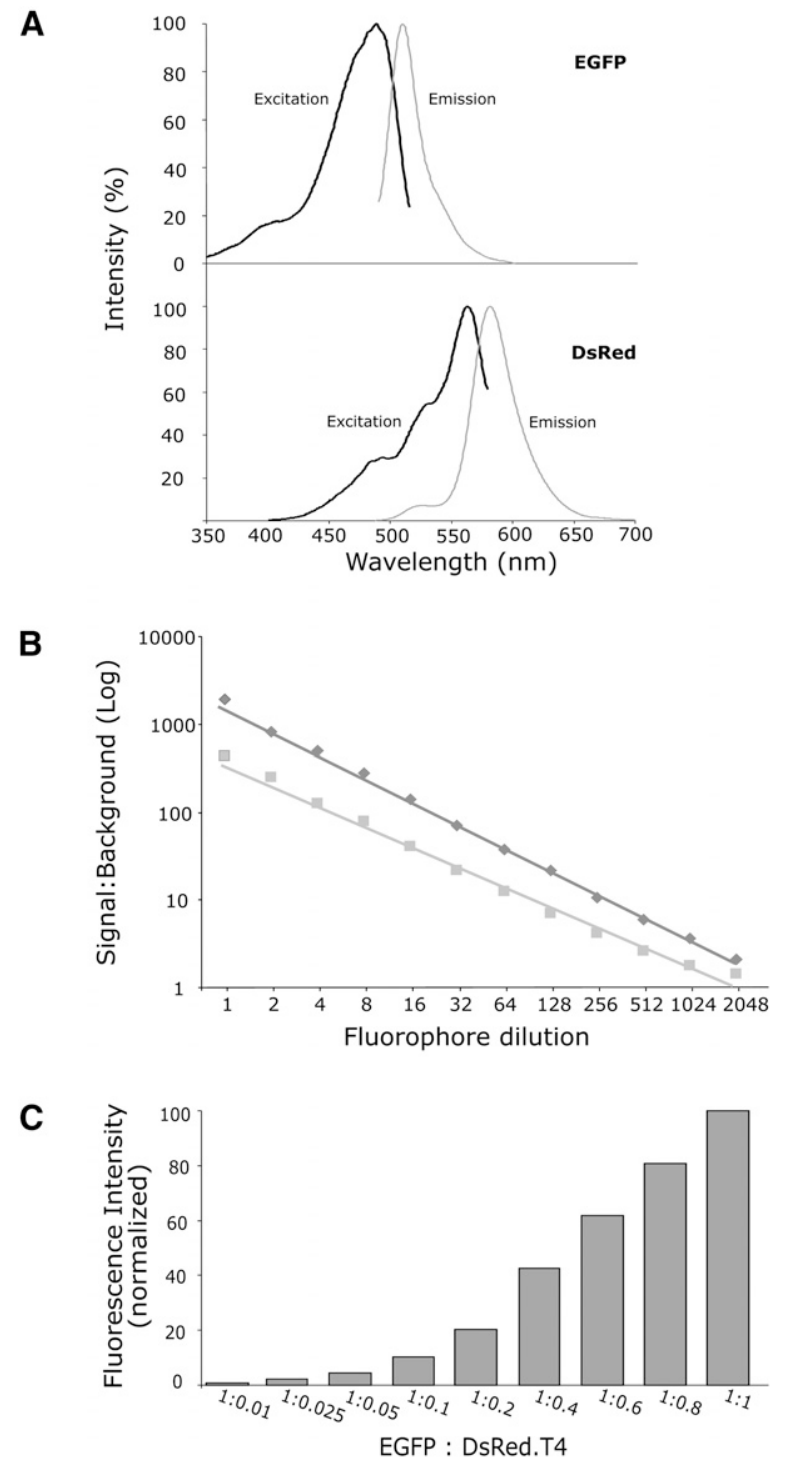

FIGURE 1. Fluorescence characterization of EGFP and DsRed.T4. (A) Normalized excitation and emission spectra of EGFP and DsRed (McNamara et al. 2006). (B) DsRed.T4 fluorescence gives a higher signal to background ratio than EGFP. Fluorophore preparations were derived from individual expressions in COS-7 cells. Dilutions were made in passive lysis buffer. The signal to background ratio was calculated from the DsRed.T4 or EGFP reading at their respective wavelengths divided by that obtained from a lysate of untransfected cells. DsRed.T4 indicated by black diamonds; EGFP, gray squares. $(C)$ DsRed.T4 can be detected independently without interference from EGFP. EGFP and DsRed.T4 were expressed separately in HEK 293T cells. Cell lysates from each expression were mixed in the proportions shown with the volume supplemented (to $100 \mu \mathrm{L}$ ) as required with lysate from untransfected cells.

increasing quantities of DsRed.T4 lysate (DsRed.T4:EGFP; 0.01:1 to 1:1) while maintaining the same final volume. The fluorescence intensity showed that the downstream reporter, DsRed.T4 (see below) that would be expressed at low levels in our bicistronic system, was detected as expected as its concentration increased within the mixture 
(Fig. 1C). This was particularly so in the $1 \%-10 \%$ range, the ratios expected for frameshift experiments.

We confirmed experimentally that DsRed.T4 was the best fluorophore for the downstream reporter using the HIV-1 frameshift sequence as a recoding element. Two constructs were tested; one where the two folded fluorophores in a bicistronic fusion protein would simply be separated by the amino acids encoded within the recoding sequence, and a second that contained an additional linker sequence encoding an $18 \AA \alpha$-helical element that would increase the spatial separation between the reporter proteins (Arai et al. 2001). There were two significant observations: first, we found that the signal to background ratio for the downstream reporter [Fig. 2A, (i)] increased significantly when DsRed.T4 rather than EFGP was in this orientation $\left({ }^{\star} P=\right.$ $\left.3.3 \times 10^{-6}\right)$; second, the linker element not only enhanced this effect $\left({ }^{* *} P=3.7 \times 10^{-4}\right)$ but enhanced the signal from the upstream reporter as well [Fig. 2A, (ii)]. The additional $18 \AA$ spatial separation of the reporter proteins presumably facilitated the folding of both upstream and downstream reporters with less interference (larger linkers gave only modest further enhancement; data not shown). Time-course experiments (data not shown) revealed that $48 \mathrm{~h}$ gave the optimal signal to background ratio for the downstream reporter in the EGFP-DsRed.T4 orientation with linker (Fig. 2A, left scale) sufficient for the rare -1 frameshift event to be measured accurately from DsRed.T4 fluorescence. While the frameshift ratio was consistent between 24 and $84 \mathrm{~h}$, the cells showed toxicity by $72 \mathrm{~h}$, and before $48 \mathrm{~h}$ the expression and folding of the fluorophores was significantly lower.

\section{The bicistronic assay can measure precisely different recoding events}

We then assessed whether the assay could detect and accurately measure different recoding events, and also in different cell types. The frameshift for human antizyme is forward (+1) rather than backward as with the HIV-1 event $(-1)$ and occurs at 20\%-30\% (Howard et al. 2001) compared with 5\%-10\% for HIV-1. Frameshift efficiency can be calculated in our assay utilizing a normalizing control construct where the reporter proteins are synthesized in a 1:1 molar ratio. Our assay recorded frameshift efficiencies at the HIV-1 and antizyme recoding elements expressed in both COS-7 and HEK 293T cells comparable to those reported elsewhere (Fig. 2B; Howard et al. 2001). More subtle read-through of stop codons was measured with two gene sequences encoding PTC mutations reported for two cases of Duchenne muscular dystrophy (DMD) (Lim and Rando 2008) and cystic fibrosis (Sermet-Gaudelus et al. 2007) diseases. For measurement of these non-programmed stop signals, the more sensitive variant of DsRed, tdTomato (Shaner et al. 2004), was introduced as the downstream fluorophore as it is nearly five times brighter than the
A
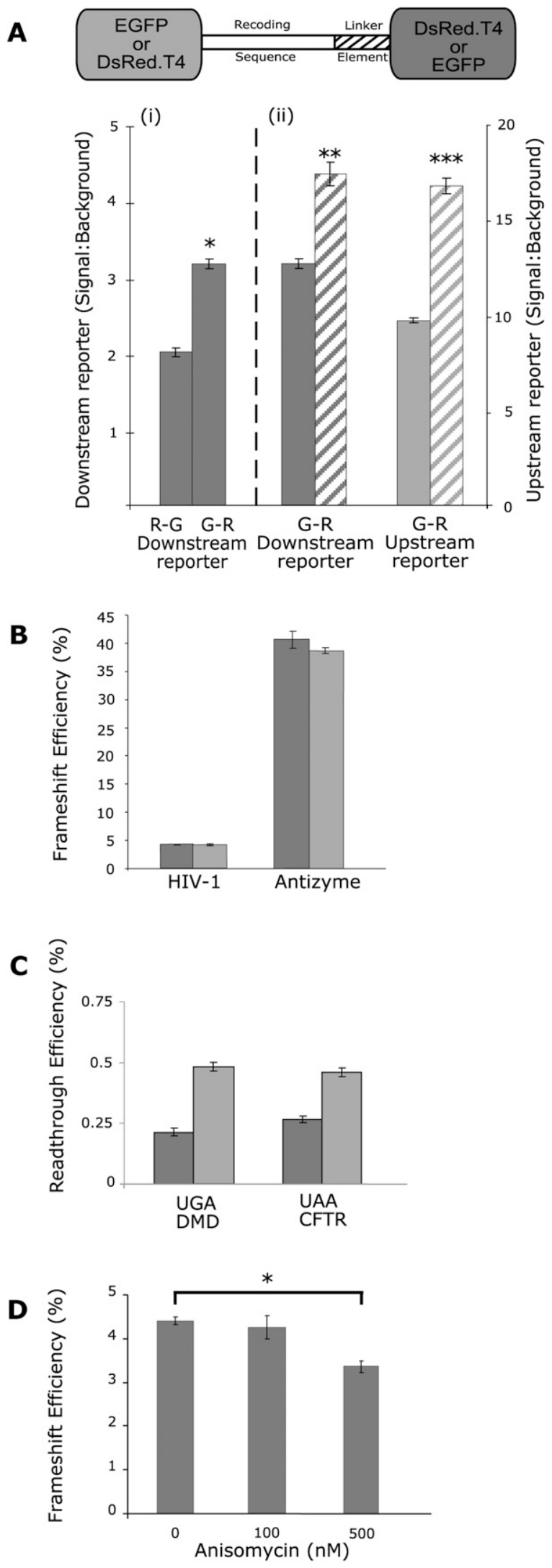

FIGURE 2. (Legend on next page) 
DsRed.T4. PTC read-through rates could be accurately determined with tight replicates for the two examples (Fig. 2C). As expected from a drug that decreases fidelity (Sermet-Gaudelus et al. 2007), gentamicin increased the read-through rate by approximately twofold in each case.

These results demonstrated the utility of the assay to record small recoding modulations such as those that might be induced by compounds during screening in a HTS assay. For proof-of-principle, we modeled increasing concentrations of anisomycin, a compound known to specifically inhibit -1 frameshifting (Dinman et al. 1997). Our fluorophore assay replicated the reported reduced frameshift rate with the HIV-1 element with this drug (Fig. 2D). Anisomycin inhibited frameshifting by $>25 \%$ at $500 \mathrm{nM}$ $(P=0.002)$ and by a lesser amount at $100 \mathrm{nM}$. Compounds that affect the overall rate of protein synthesis can be identified by changes in fluorescence measurements from the upstream reporter in our assay, whereas the recoding event is assessed from the downstream reporter. In these experiments, anisomycin started to decrease overall protein synthesis at $200 \mathrm{nM}$ and significantly by $400 \mathrm{nM}$ (data not shown). Determination of frameshift efficiency, however, normalizes for this effect.

\section{The in vivo bicistronic fluorescence assay gives comparable readings to the in vitro dual luciferase assay}

Of particular interest was to compare our new cell-based assay with the commonly used in vitro dual luciferase assay. The fluorescence and dual luciferase assays gave comparable relative results when measuring "natural" antizyme or

FIGURE 2. Detecting recoding changes using the HIV-1 frameshift element. (A) (i) DsRed.T4 as the downstream reporter gives significantly higher enhancement over background compared with EGFP $\left({ }^{*} \mathrm{P}=3.3 \times 10^{-6}\right)$. (ii) This is amplified further when a linker is included (downstream reporter ${ }^{\star *} P=3.7 \times 10^{-4}$; upstream reporter ${ }^{\star * *}$ $\left.P=3.7 \times 10^{-6}\right)$. Results show the mean and standard deviations for four replicates in COS-7 cells. Hashed bars indicate linker included; $\mathrm{R}-\mathrm{G}$, red-green; and G-R, green-red. (B) The assay is consistent in different cell types and with distinct recoding mechanisms. HEK 293T cells (dark bars) and COS-7 cells (light bars) were transfected with the bifluorophore vector containing the HIV-1 element, or the frameshift element of human antizyme. Results show the mean and standard deviations for four (HEK 293T) or eight (COS-7) cell replicates. (C) Read-through rates of premature termination codons. With tdTomato as the downstream fluorophore, the assay measured small changes in read-through, and increased translational infidelity with gentamicin. Known nonsense mutations in the DMD (UGA) and CFTR (UAA) genes were inserted into the bifluorophore vector and expressed in HEK 293T cells with (light bars) or without (dark bars) gentamicin $(600 \mu \mathrm{g} / \mathrm{mL})$ (Matthews et al. 1977; Srikantha et al. 1996). Results show the mean and standard deviations for eight replicates. DMD indicates Duchenne muscular dystrophy; CFTR, cystic fibrosis transmembrane receptor. $(D)$ Changes in frameshifting at the HIV-1 element induced by a drug such as anisomycin can be measured. Transfected COS-7 cells were incubated with anisomycin for $48 \mathrm{~h}$. Results show the mean and standard deviations for 12 replicates.
HIV-1 frameshift events (Fig. 3A). Frameshifting was high with both bicistronic reporter sets for antizyme $(\sim 40 \%-$ $47 \%)$ and much lower for HIV-1 ( $\sim 4 \%-10 \%)$. The two assays did not give identical absolute efficiency numbers; $\sim 10 \%$ for HIV-1 (dual luciferase) compared with $\sim 4 \%$ (bifluorophore). Nevertheless, both assays detected subtle changes in frameshift efficiency arising from induced mutations in the HIV-1 element that disrupted the base of the secondary structural stem-loop (GGG codon altered to GGA) (dual luciferase: $9.2 \%$ reduced to $7.4 \%$; bifluorophore: $5.7 \%$ reduced to $4.7 \%$ ) (Fig. 3B). A mutation that has a stronger disruptive effect (GGG to UGA) was recorded similarly in both assays (2.8-3.2\%). Likewise, both assays consistently measured very low efficiency recoding events resulting from read-through of PTC mutations in DMD and CF (Fig. 3C). In these cases, the efficiencies were reproducibly consistent within each assay and were replicated faithfully in different transfections on different days.

\section{The bicistronic fluorophore assay can be used in a 96-well plate format}

We designed this sensitive bicistronic fluorophore system as a HTS assay for identifying lead compounds that affect genetic recoding. The development studies shown here used a 24-well plate format and lysed cells with $Z^{\prime}$-factors, reflecting both the scatter in the data and signal to background ratios (Zhang et al. 1999), indicating it rated as an excellent assay (Table 1). In contrast, the sensitive dual luciferase assay gives outlier readings more frequently in both our hands and others (Jacobs and Dinman 2004) that adversely affect the $Z^{\prime}$-factor. Similarly, measuring whole nonlysed cells with our detector that samples only a fraction of the well also compromised the $\mathrm{Z}^{\prime}$-factor (data not shown). Cell lysis, however, may not be necessary for a detector that measures an entire well. We extended our assay to a 96-well plate format and found the DsRed.T4 fluorophore began to fail for detection of the low frequency recoding event, indicated by the low signal to background ratio and frameshift efficiency (Table 1). When the more sensitive tdTomato fluorophore replaced DsRed.T4, frameshift efficiency changes comparable with the 24-well plate were restored and the $Z^{\prime}$-factor again rated the assay as excellent (Table 1).

\section{Does the identity of the downstream red fluorophore affect the assay?}

We anticipated that the two bicistronic systems based on different reporters might not give the same absolute recoding efficiencies. Indeed, that was the case as shown for the fluorophore and luciferase systems (see Fig. 3). Upstream reporters produced without a C-terminal downstream reporter (the dominant event) may fold more easily than a fusion protein (the recoding event), and this is likely to 
A

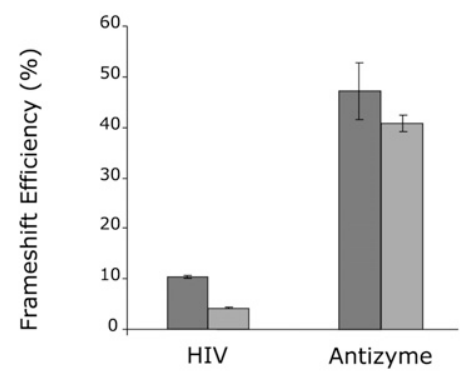

B

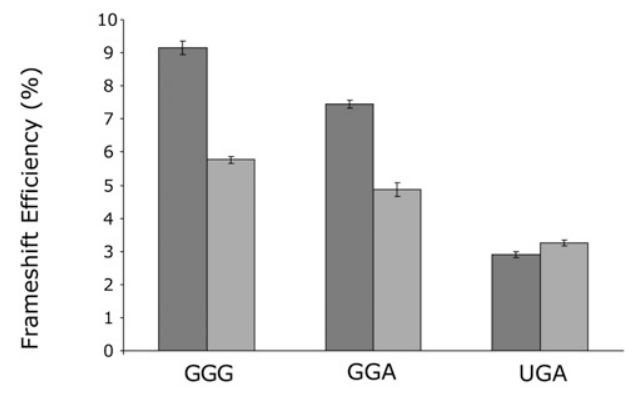

C

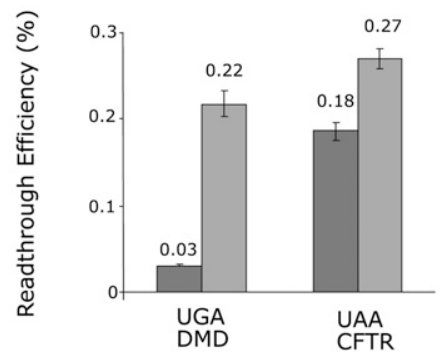

FIGURE 3. The bifluorophore and dual luciferase assays give comparable recoding efficiencies. (A) Constructs containing the HIV-1 or the antizyme +1 frameshift elements or controls were expressed in HEK 293T cells. Results show the mean and standard deviations for four or more replicates. (B) Detection of small frameshift efficiency changes. Test constructs containing the native (GGG) stem-loop and alterations that disrupt the bottom of the stem-loop (GGA, UGA) and controls were expressed in COS-7 cells for both assays. Results show the mean and standard deviations for three or more replicates. $(C)$ Detection of low-level read-through at premature termination codon sites. The constructs containing the PTC mutations and controls were expressed in HEK 293T cells for both assays. Results show the mean and standard deviations for eight or more replicates. Dual luciferase assay indicated by dark bars; bifluorophore assay, light bars.

vary among different reporters. We compared different downstream "red" fluorophores to find out whether within the bicistronic fusion protein the protein folding or the molecular arrangement required for fluorescence (Table 2) influenced the calculated frameshift efficiency of the upstream test HIV-1 recoding element (Fig. 4). A significant observation emerged: when monomeric mCherry (Shaner et al. 2004) (another variant of DsRed) which does not require oligomerization was used as the downstream reporter, the calculated frameshift rate was $6.8 \%$, that is higher than that of tdTomato (5.5\%) (two units in cis) which folds and then "self-dimerizes" for activity. In turn, this value was significantly greater than for the dTomato alone (4.4\%) that must form dimers in trans from different fusion protein molecules. The tetrameric DsRed.T4 requiring four subunits in trans gave the lowest value. Although mCherry gave the highest frameshift efficiency and has a similar relative brightness to DsRed.T4 (Table 2), it has more red-shifted excitation and emission and requires specific filters; its signal was at the detection limit with the filter sets from which we developed the fluorophore assay.

\section{DISCUSSION}

We have developed a simple homogeneous bifluorophore assay that can be adapted for any recoding study by inserting a sequence element of interest between the modular flanking reporter genes. This is the first report describing a mammalian cell-based assay for recoding biology using fluorescent bicistronic reporters. It is amenable to HTS and drug discovery and has the potential for multiplexing with other markers in vivo. The use of fluorescent reporters circumvents the extra steps needed for an enzyme assay and consequently decreases the read time of a 96-well plate $\sim 10$-fold. With no need for the expensive substrates required for the luciferase reporters, there can be a significant cost saving in large screens. In our 24- and 96-well formats, the cost was reduced by $\sim 90 \%$. Key scientific benefits of the assay were the low scatter of replicates in experiments and the day-to-day reproducibility of test samples. This feature of the fluorophore assay compensated for it not having the intrinsic signal amplification of the enzyme assay and meant very small changes in recoding efficiencies could be accurately monitored. The orientation of the fluorescent reporters and addition of a linker element significantly enhanced the assay, increasing the signal above the background noise. A Z'-factor indicative of a high quality assay was obtained even for low signal to noise ratios.

Further miniaturization (384-wells) utilizing state-ofthe-art fluorescence plate readers and appropriate fluorophore and filter set combinations is feasible and would decrease cost even further. Our studies suggest that the more "red-shifted" mCherry variant might be an excellent downstream fluorophore. This could further enhance maximal excitation and emission for the downstream reporter without interference from the upstream EGFP reporter or

TABLE 1. Comparison of DsRed.T4 and tdTomato in 24-well and 96-well plate formats

\begin{tabular}{|c|c|c|c|c|}
\hline \multirow{2}{*}{$\begin{array}{l}\text { Assay format } \\
\text { (3' fluorophore) }\end{array}$} & \multicolumn{2}{|c|}{ 24-Well } & \multicolumn{2}{|c|}{ 96-Well } \\
\hline & DsRed.T4 & tdTomato & DsRed.T4 & tdTomato \\
\hline Signal:background & 10.4 & 32.2 & 2.9 & 6.6 \\
\hline $\begin{array}{l}\text { Frameshift } \\
\text { efficiency (\%) }\end{array}$ & 4.2 & 5.3 & 2.1 & 5.2 \\
\hline$Z^{\prime}$-factor & 0.6 & 0.8 & 0.6 & 0.8 \\
\hline
\end{tabular}


TABLE 2. Comparison of the fluorescent characteristics of DsRed fluorophore variants

\begin{tabular}{lcc}
\hline Fluorophore & $\begin{array}{c}\text { Molecular } \\
\text { arrangement required } \\
\text { for fluorescence }\end{array}$ & $\begin{array}{c}\text { Brightness } \\
\text { of mature protein } \\
\text { (relative to DsRed.T4) }\end{array}$ \\
\hline DsRed.T4 & Tetramer & 1.0 \\
dTomato & Dimer & 2.4 \\
tdTomato & Dimer $^{\mathrm{b}}$ & 4.8 \\
mCherry & Monomer & 0.8 \\
\hline
\end{tabular}

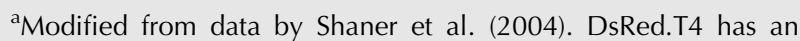
equivalent profile to DsRed.T1.

${ }^{\mathrm{b}}$ The in cis molecules dimerize.

any naturally fluorescent compounds tested in HTS. However, we found the tdTomato reporter provided the necessary signal to background ratio in this development phase for detecting very subtle recoding changes.

This assay was developed to detect changes in the measured translational parameters, frameshifting or read-through, ideally in a drug screen. It proved very effective at measuring differences as low as $0.1 \%$ reproducibly both within an experiment and between experiments (see Fig. 2). While absolute efficiencies of the recoding event measured with different reporters might vary, this would not be a significant issue to using the assay where changes are being assessed between two conditions. Indeed, a comparison between luciferase and fluorophore reporters showed that although the absolute measured values were slightly different, the subtle changes made to the sequence element under study were reflected in the results of both reporter sets (see Fig. 3).

Additionally, because this system relies on both reporter proteins to fold correctly, and, in some cases, to form functional oligomeric complexes, there was potential for slow development of a functional unit or misfolding to affect the fluorescent signal output from a specific reporter. Use of different downstream fluorophore reporters that each had red excitation and emission wavelengths was informative. We found a range of frameshift efficiencies from $4 \%-7 \%$ at the HIV-1 element. The mCherry reporter, which relies on simple folding of a single domain for its fluorescent output, gave the highest apparent efficiency for frameshifting. The dTomato reporter with a lower measured efficiency requires formation of a dimer in trans (or in cis in the tdTomato), suggesting that there was a lower efficiency of folding/dimerizing to give an active domain than for mCherry, and that this was more difficult to achieve in trans. The DsRed.T4 requires a tetramer for an active fluorescing domain, and this must form from four different molecules (in our case, fusion proteins). Not surprisingly, this gave the lowest efficiency for the recoding event of the set tested (see Fig. 4). While these influences could compromise a particular downstream reporter, each of those tested is still suitable for the developed assay, providing the signal to background ratio of the downstream reporter is sufficient to give a high $\mathrm{Z}^{\prime}$-factor.

Genetic recoding is predominantly a viral mechanism, and therefore, targeting such events for drug development has high potential. The assay we have developed is useful for HTS for this purpose. Nonprogrammed stop codons that are introduced into human genes by nonsense mutations create stop signals of different efficiencies and have individual potential to be modulated by drugs that affect translational fidelity (the ability to incorporate an amino acid at translational stops can be strongly influenced by context, with the stop signal having a core of four bases within a larger sequence element). Our assay can both assess the potential of these nonsense mutation contexts for modulation and act as an assay for screening compounds that affect such fidelity.

\section{MATERIALS AND METHODS}

\section{Construction of bicistronic reporter vectors}

Standard cloning and PCR mutagenesis procedures were used to construct modular dual reporter vectors by adapting pDsRed.T4N1 (HIV series and antizyme) (Shaner et al. 2004) and pcDNA5/ FRT (Invitrogen: EGFP-tdTomato; CFTR and DMD series), to enable two fluorophores to be inserted, with a cloning site, signal sequence, and additional linker element (for sequences, see Supplemental Table).

\section{Luciferase vectors}

Annealed oligonucleotides were ligated ( $5^{\prime}$ EcoRI/3' SacII) into the bicistronic reporter vector pGL3s-hRLuc ${ }^{+}$(previously adapted from pGL3-Control [Promega] to include the human codon optimised hRluc gene upstream, a downstream $L u c+$ gene, and the SacII cloning site).

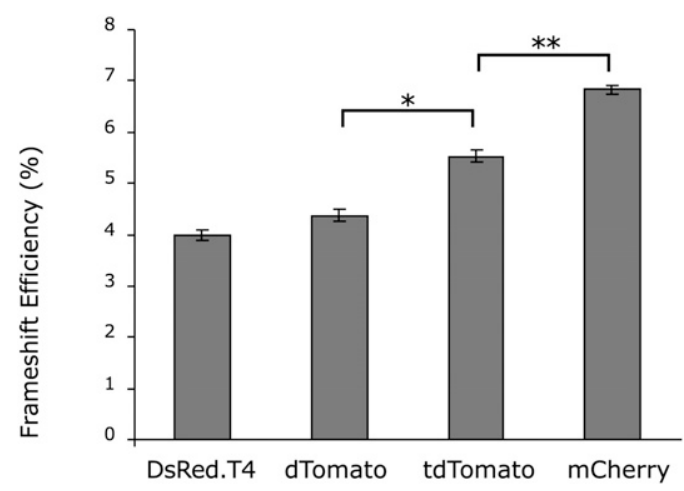

FIGURE 4. The influence of the formation of the 3 ' reporter fluorophore on the measured frameshift efficiency. Constructs containing the HIV-1 frameshift element or controls were expressed in HEK 293T cells. The downstream reporters with excitation and emission in the red part of the spectrum were mCherry, dTomato, tdTomato, and DsRed.T4. Results show the mean and standard deviations for 12 replicates. tdTomato gave a frameshifting efficiency statistically different from dTomato $\left({ }^{\star} P=0.003\right.$; and DsRedT4) and mCherry $\left({ }^{\star \star} P=0.001\right)$. 


\section{Bicistronic fluorophore vectors}

A modified EGFP (pEGFP-N1, BD Biosciences) containing a $5^{\prime}$ HindIII site and an EcoRI site in place of the $3^{\prime}$ stop codon (primers: EGFP-f, -r) was inserted into pDsRed.T4-N1. The DsRed.T4 gene was replaced with a modified gene containing a BamH1 site in place of the start codon (primers: DsRed.T4-f, -r; pEGFP-DsRed.T4). Similarly, a vector with the reporters in the reverse order was made by sequentially removing each fluorescent gene from pEGFP-DsRed.T4 and inserting the other fluorescent gene with compatible restriction sites added (primers: Inv.EGFP-f, -r; Inv.DsRed.T4-f, -r) (pDsRed.T4-EGFP)

\section{Linker insertion}

A synthetic linker encoding EAAAKEAAAKA was added immediately $5^{\prime}$ to the downstream reporter using annealed oligonucleotides (5' SacII/3' BamH1). The oligonucleotides were designed to ensure the repeating amino acids used different codons (Linker-f, -r)

\section{Recoding elements}

Test and control HIV sequence elements were created by insertion of annealed oligonucleotides ( $5^{\prime}$ EcoR1/3' SacII) between the fluorescence reporters (oligonucleotides: HIV-f, -r; HIV null 0-f, -r). The HIV constructs all contained the heptanucleotide "slippery" sequence immediately followed by GGG and the extended stemloop (Dulude et al. 2002). The 0-frame construct (equimolar amounts of each reporter protein produced for normalization of individual signal outputs from the two reporters) contained the "slippery" sequence UUUUUUAGGG replaced with UUUCCUA GGG encoding the same amino acids. An additional $\underline{A}$ was inserted immediately $5^{\prime}$ of the GGG to place the downstream reporter in the 0 -frame. In addition, HIV constructs were made where the wild-type GGG codon promoting frameshifting was modified to GGA or replaced with the stop codon UGA. The human antizyme frameshift sequence (see Supplemental Table) was inserted as a +1 frameshift element. Annealed oligonucleotides containing the CFTR (Y122X) (Sermet-Gaudelus et al. 2007) and DMD (S319X) (Bidou et al. 2004) stop codon read-through sequences (GFPCFTR-f, -r; GFPDMD-f, -r) were inserted into the dual GFP vector pEGFP-DsRed.T4 as above, then the entire EGFP/ test sequence/linker element excised (HindIII/BamHI), and ligated in place of the corresponding sequence upstream of the tdTomato reporter in pcDNA5/FRT

\section{tdTomato, dTomato, and mCherry fluorophore substitutions}

The pcDNA5/FRT vector was adapted to incorporate the EGFP and tdTomato (Shaner et al. 2004), dTomato or mCherry fluorophores. The EGFP-DsRed.T4 reporter genes with the test sequences and linker element were inserted into pcDNA5/FRT (HindIII/Not1); then the DsRed.T4 gene was removed (BamHI/ NotI) and replaced with the tdTomato, dTomato, or mCherry gene containing a BamH1 site in place of the start codon (Fruit-f, -r).

\section{Cell culture and cell lysate preparation}

COS-7 or HEK 293T cells were transiently transfected with $500 \mathrm{ng}$ of the dual fluorophore or $250 \mathrm{ng}$ of the luciferase reporter vector using a 4:1 ratio of FuGENE 6 Transfection Reagent (Invitrogen) to DNA, added either at the time of seeding $\left(1 \times 10^{5}\right.$ cells $\left./ \mathrm{mL}\right)$, or
24-36 h after seeding $\left(2 \times 10^{4}\right.$ cells/mL: for the luciferase experiments shown in Figure 3B), into clear 24-well or black 96-well cell culture plates (Greiner Bio-One). Cells were grown for $48 \mathrm{~h}\left(37^{\circ} \mathrm{C}\right.$ and $5 \% \mathrm{CO}_{2}$ ), washed with PBS (to remove phenol red that interferes with detection measurements), lysed in $100 \mu \mathrm{L}$ Passive Lysis Buffer (Promega) on a shaking tray (Stuart Scientific Platform STR8) at RT for $15 \mathrm{~min}$, and then frozen for later analysis.

\section{Fluorophore detection}

To verify the excitation and emission maxima of the fluorophores, cell lysates were transferred into glass cuvettes for detection in a Varian Cary Eclipse Fluorescence spectrophotometer (Varian). For all other fluorophore detections, cell lysates were transferred into black 96-well plates (Greiner Bio-One) and measured using a BMG POLARstar optima (BMG LABTECH). EGFP fluorescence was detected using 485-nm excitation/520-nm emission spectra, while DsRed.T4, dTomato, tdTomato, and mCherry fluorophores were detected using 544-nm excitation/590-nm emission spectra.

\section{Luminescence detection}

The Luc+ assay was performed as described (Tanguay and Gallie 1996) and RLuc luciferase assay used the conditions as described (Matthews et al. 1977) with modifications (Srikantha et al. 1996). Luciferase activities in cell lysates were determined by adding 50 $\mu \mathrm{L}$ of the corresponding assay buffer for RLuc or Luc+ to between $1 \mu \mathrm{L}$ and $10 \mu \mathrm{L}$ of lysate. Luminescence was measured immediately after the addition of the assay buffer using an Auto Lumat LB953 luminometer (Berthold Technologies) or a BMG POLARstar optima (BMG LABTECH).

\section{Calculation of frameshift and read-through efficiencies}

The relative fluorescence units (RFU) (fluorophore assay) and the relative light units (RLU) (luciferase assay) were corrected for the respective background fluorescence or luminescence readings. The corrected RFUs or RLUs were used to calculate the ratios of the downstream:upstream reporters for the test constructs and control constructs (where equimolar ratios of the two products are synthesized). Frameshift or read-through efficiencies (\%) = (test construct ratio/comparable null 0 -frame construct ratio) $\times$ 100. The Student's two-tailed $t$-test assuming equal variance was used to calculate $P$-values after consultation with a biostatistics informatician. Western analysis confirmed synthesis of EGFP and the EGFP-DsRed.T4 fusion protein at the expected ratios, with the fusion protein just detectable.

\section{$Z^{\prime}$-factor calculation}

The $Z^{\prime}$-factor provides a tool for comparing and evaluating the quality of an assay (Zhang et al. 1999). A $Z^{\prime}$-factor of $\geq 0.5$ but $<1$ represents an excellent assay. The $Z^{\prime}$-factor $=1-(3 \times \mathrm{SD}$ of the downstream reporter $+3 \times \mathrm{SD}$ of cell-only lysate control) $/($ mean of the downstream reporter-mean of the cell-only lysate control). $\mathrm{SD}$ indicates standard deviation. $\mathrm{Z}^{\prime}$-factors were also calculated for the upstream reporters.

\section{SUPPLEMENTAL DATA}

Supplemental material can be found at http://www.rnajournal.org. 


\section{POTENTIAL CONFLICT OF INTEREST DISCLOSURE}

T.S.C. and W.P.T. hold Patent WO/2007/027106 "Dual fluorescent reporter construct and assay for measuring translational recoding."

\section{ACKNOWLEDGMENTS}

This work was supported by grants from the Health Research Council of New Zealand (grant Nos. 01/317, 05/195 to W.P.T. and E.S.P) and a University of Otago Postgraduate Scholarship to S.F.M. We thank Ben Glick (University of Chicago) for the kind gift of pDsRed.T4-N1; and Roger Tsien (University of California, San Diego) for the kind gift of the dTomato, tdTomato, and mCherry vectors; and Mik Black (University of Otago) for biostatistical advice.

Received February 4, 2009; accepted April 24, 2009.

\section{REFERENCES}

Arai R, Ueda H, Kitayama A, Kamiya N, Nagamune T. 2001. Design of the linkers which effectively separate domains of a bifunctional fusion protein. Protein Eng 14: 529-532.

Bertram G, Innes S, Minella O, Richardson JP, Stansfield I. 2001. Endless possibilities: Translation termination and stop codon recognition. Microbiology 147: 255-269.

Bevis BJ, Glick BS. 2002. Rapidly maturing variants of the Discosoma red fluorescent protein. Nat Biotechnol 20: 83-87.

Bidou L, Hatin I, Perez N, Allamand V, Panthier JJ, Rousset JP. 2004. Premature stop codons involved in muscular dystrophies show a broad spectrum of readthrough efficiencies in response to gentamicin treatment. Gene Ther 11: 619-627.

Biswas P, Jiang X, Pacchia AL, Dougherty JP, Peltz SW. 2004. The human immunodeficiency virus type 1 ribosomal frameshifting site is an invariant sequence determinant and an important target for antiviral therapy. J Virol 78: 2082-2087.

Dinman JD, Ruiz-Echevarria MJ, Czaplinski K, Peltz SW. 1997. Peptidyl-transferase inhibitors have antiviral properties by altering programmed -1 ribosomal frameshifting efficiencies: Development of model systems. Proc Natl Acad Sci 94: 6606-6611.

Dos Ramos F, Carrasco M, Doyle T, Brierley I. 2004. Programmed -1 ribosomal frameshifting in the SARS coronavirus. Biochem Soc Trans 32: 1081-1083.

Dulude D, Baril M, Brakier-Gingras L. 2002. Characterization of the frameshift stimulatory signal controlling a programmed -1 ribosomal frameshift in the human immunodeficiency virus type 1 . Nucleic Acids Res 30: 5094-5102.

Dulude D, Théberge-Julien G, Brakier-Gingras L, Heveker N. 2008. Selection of peptides interfering with a ribosomal frameshift in the human immunodeficiency virus type 1. RNA 14: 981-991.

Farabaugh PJ. 1996. Programmed translational frameshifting. Annu Rev Genet 30: 507-528.

Gesteland RF, Weiss RB, Atkins JF. 1992. Recoding: Reprogrammed genetic decoding. Science 257: 1640-1641.
Grentzmann G, Ingram JA, Kelly PJ, Gesteland RF, Atkins JF. 1998. A dual-luciferase reporter system for studying recoding signals. RNA 4: 479-486.

Harger JW, Dinman JD. 2003. An in vivo dual-luciferase assay system for studying translational recoding in the yeast Saccharomyces cerevisiae. RNA 9: 1019-1024.

Howard MT, Shirts BH, Zhou JD, Carlson CL, Matsufuji S, Gesteland RF, Weeks RS, Atkins JF. 2001. Cell culture analysis of the regulatory frameshift event required for the expression of mammalian antizymes. Genes Cells 6: 931-941.

Jacobs JL, Dinman JD. 2004. Systematic analysis of bicistronic reporter assay data. Nucleic Acids Res 32: e160.

Jacobs JL, Belew AT, Rakauskaite R, Dinman JD. 2007. Identification of functional, endogenous programmed -1 ribosomal frameshift signals in the genome of Saccharomyces cerevisiae. Nucleic Acids Res 35: $165-174$.

Kellermayer R. 2006. Translational readthrough induction of pathogenic nonsense mutations. Eur J Med Genet 49: 445-450.

Liao P-Y, Gupta P, Petrov AN, Dinman JD, Lee KH. 2008. A new kinetic model reveals the synergistic effect of E-, P- and A-sites on +1 ribosomal frameshifting. Nucleic Acids Res 36: 2619-2629.

Lim LE, Rando TA. 2008. Technology insight: Therapy for Duchenne muscular dystrophy-An opportunity for personalized medicine? Nat Clin Pract Neurol 4: 149-158.

Matthews JC, Hori K, Cormier MJ. 1977. Purification and properties of Renilla reniformis luciferase. Biochemistry 16: 85-91.

McNamara G, Gupta A, Reynaert J, Coates TD, Boswell K. 2006. Spectral imaging microscopy web sites and data. Cytometry A 69: 863-871.

Poole ES, Major LL, Cridge AG, Tate WP. 2004. The mechanism of recoding in pro- and eukaryotes. In Protein synthesis and ribosome structure (eds. K Nierhaus and DN Wilson), pp. 397-428. Wiley$\mathrm{VCH}$, Weinheim, Germany.

Sermet-Gaudelus I, Renouil M, Fajac A, Bidou L, Parbaille B, Pierrot S, Davy N, Bismuth E, Reinert P, Lenoir G, et al. 2007. In vitro prediction of stop-codon suppression by intravenous gentamicin in patients with cystic fibrosis: A pilot study. BMC Med 5: 5 .

Shaner NC, Campbell RE, Steinbach PA, Giepmans BNG, Palmer AE, Tsien RY. 2004. Improved monomeric red, orange, and yellow fluorescent proteins derived from Discosoma sp. red fluorescent protein. Nat Biotechnol 22: 1567-1572.

Shehu-Xhilaga M, Crowe SM, Mak J. 2001. Maintenance of the Gag/ Gag-Pol ratio is important for human immunodeficiency virus type 1 RNA dimerization and viral infectivity. J Virol 75: 1834 1841.

Small-Howard AL, Berry MJ. 2005. Unique features of selenocysteine incorporation function within the context of general eukaryotic translational processes. Biochem Soc Trans 33: 1493-1497.

Srikantha T, Klapach A, Lorenz WW, Tsai LK, Laughlin LA, Gorman JA, Soll DR. 1996. The sea pansy Renilla reniformis luciferase serves as a sensitive bioluminescent reporter for differential gene expression in Candida albicans. J Bacteriol 178: 121129.

Tanguay RL, Gallie DR. 1996. Translational efficiency is regulated by the length of the 3' untranslated region. Mol Cell Biol 16: 146156.

Zhang JH, Chung TD, Oldenburg KR. 1999. A simple statistical parameter for use in evaluation and validation of high throughput screening assays. J Biomol Screen 4: 67-73. 

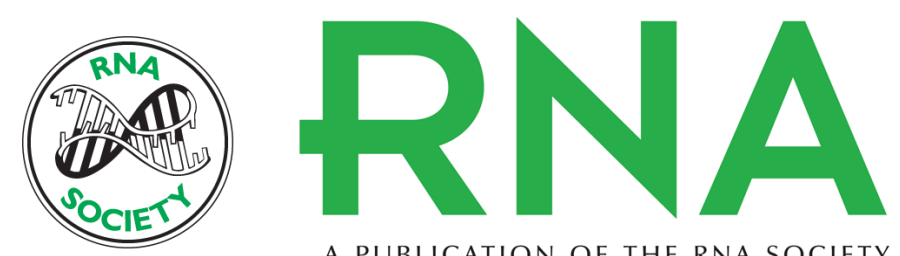

A PUBLICATION OF THE RNA SOCIETY

\section{A homogeneous cell-based bicistronic fluorescence assay for high-throughput identification of drugs that perturb viral gene recoding and read-through of nonsense stop codons}

Tony S. Cardno, Elizabeth S. Poole, Suneeth F. Mathew, et al.

RNA 2009 15: 1614-1621 originally published online June 17, 2009

Access the most recent version at doi:10.1261/rna.1586709

Supplemental Material

References

Open Access

License Freely available online through the RNA Open Access option.

Email Alerting Service
http://rnajournal.cshlp.org/content/suppl/2009/06/19/rna.1586709.DC1

This article cites 28 articles, 9 of which can be accessed free at: http://rnajournal.cshlp.org/content/15/8/1614.full.html\#ref-list-1

Freely available online through the RNA Open Access option.

top right corner of the article or click here. 